\title{
TXS 0506+056, the first cosmic neutrino source, is not a BL Lac
}

\author{
P. Padovani ${ }^{1,2 \star}$, F. Oikonomou ${ }^{1}$, M. Petropoulou ${ }^{3}$, P. Giommi ${ }^{4,5,6}$, E. Resconi ${ }^{7}$ \\ ${ }^{1}$ European Southern Observatory, Karl-Schwarzschild-Str. 2, D-85748 Garching bei München, Germany \\ ${ }^{2}$ Associated to INAF - Osservatorio Astronomico di Roma, via Frascati 33, I-00040 Monteporzio Catone, Italy \\ ${ }^{3}$ Department of Astrophysical Sciences, Princeton University, New Jersey 08544, USA \\ ${ }^{4}$ Agenzia Spaziale Italiana, ASI, via del Politecnico s.n.c., I-00133 Roma Italy \\ ${ }^{5}$ Institute for Advanced Studies, Technische Universität München, Lichtenbergstrasse 2a, D-85748 Garching bei München, Germany \\ ${ }^{6}$ ICRANet, Piazzale della Repubblica 10, I-65122, Pescara, Italy \\ ${ }^{7}$ Technische Universität München, Physik-Department, James-Frank-Str. 1, D-85748 Garching bei München, Germany
}

Accepted 2019 January 19. Received 2019 January 15; in original form 2018 September 21

\begin{abstract}
We present evidence that TXS $0506+056$, the first plausible non-stellar neutrino source, despite appearances, is not a blazar of the BL Lac type but is instead a masquerading BL Lac, i.e., intrinsically a flat-spectrum radio quasar with hidden broad lines and a standard accretion disk. This re-classification is based on: (1) its radio and O II luminosities; (2) its emission line ratios; (3) its Eddington ratio. We also point out that the synchrotron peak frequency of TXS $0506+056$ is more than two orders of magnitude larger than expected by the so-called "blazar sequence", a scenario which has been assumed by some theoretical models predicting neutrino (and cosmic-ray) emission from blazars. Finally, we comment on the theoretical implications this reclassification has on the location of the $\gamma$-ray emitting region and our understanding of neutrino emission in blazars.
\end{abstract}

Key words: neutrinos — radiation mechanisms: non-thermal — galaxies: active BL Lacertae objects: general - gamma-rays: galaxies

\section{INTRODUCTION}

The IceCube Collaboration together with the multimessenger community has recently reported on the association of high-energy neutrinos with the blazar TXS 0506+056 $(z=0.3365)$. This has been triggered by the detection of a muon neutrino with most probable energy $\sim 290 \mathrm{TeV}$ from the direction of the blazar at the $3-3.5 \sigma$ level (IceCube Collaboration et al. 2018). Fermi-LAT observations have revealed that TXS 0506+056 was in a flaring state at the time of the IceCube alert, with a $0.1-300 \mathrm{GeV}$ flux higher by a factor $\sim 6$ than the average reported in the 3LAC catalogue (Ackermann et al. 2015; IceCube Collaboration et al. 2018). Follow-up observations have led to the detection of the source with the MAGIC and VERITAS telescopes at energies $>100 \mathrm{GeV}$, as well as in the X-ray, optical, and radio bands, by Swift/XRT, NuSTAR, ASAS-SN, VLA, and various other facilities (IceCube Collaboration et al. 2018; Abeysekara et al. 2018; Albert et al. 2018; Ansoldi et al. 2018). Subsequent analysis of archival IceCube data has revealed $13 \pm 5$ muon neutrinos in excess of background ex-

^ E-mail: ppadovan@eso.org pectations arriving from the same region of the sky on a time-scale of $4-5$ months in 2014-2015, which constitutes $3.5 \sigma$ evidence for neutrino emission from the direction of TXS 0506+056 (IceCube Collaboration 2018). A dissection of the region around the neutrino position has further shown that TXS $0506+056$ is the only counterpart of all the neutrino emission in the region and therefore the most plausible first high-energy neutrino source (Padovani et al. 2018).

From the optical spectroscopy viewpoint blazars are historically divided in two classes, namely flat-spectrum radio quasars (FSRQs) and BL Lac objects (henceforth, BL Lacs), with the former displaying strong, broad emission lines just like standard quasars, and the latter instead showing at most weak emission lines, sometimes exhibiting absorption features, and in many cases being completely featureless (Urry \& Padovani 1995). Notably, all FSRQs are of the low-energy peaked $\left(\mathrm{LBL}^{1}\right)$, and in a few cases, IBL type.

\footnotetext{
1 Blazars are divided based on the rest-frame frequency of the low-energy (synchrotron) hump $\left(v_{\text {peak }}^{S}\right)$ into LBL sources $\left(v_{\text {peak }}^{S}<\right.$ $\left.10^{14} \mathrm{~Hz}[<0.41 \mathrm{eV}]\right)$, intermediate- $\left(10^{14} \mathrm{~Hz}<v_{\text {peak }}^{S}<10^{15} \mathrm{~Hz}[0.41\right.$ $\mathrm{eV}-4.1 \mathrm{eV})]$, and high-energy $\left(v_{\text {peak }}^{\mathrm{S}}>10^{15} \mathrm{~Hz}[>4.1 \mathrm{eV}]\right)$ peaked
} 
In this Letter we show that TXS $0506+056$ is not what it looks like, i.e., a blazar of the BL Lac type, but instead is intrinsically an FSRQ. We also briefly comment on the implications this might have on the theoretical modeling of this source. We use a $\Lambda$ CDM cosmology with $H_{0}=70 \mathrm{~km}$ $\mathrm{s}^{-1} \mathrm{Mpc}^{-1}, \Omega_{\mathrm{m}, 0}=0.3$, and $\Omega_{\Lambda, 0}=0.7$.

\section{THE NATURE OF TXS 0506+056}

The first clue about the nature of TXS 0506+056 comes from its spectral energy distribution (SED), which shows the double-humped structure typical of blazars (e.g. Padovani et al. 2017) with a $v_{\text {peak }}^{S} \lesssim 10^{15} \mathrm{~Hz}$, which puts it in the IBL/HBL transition region (see, e.g., Fig. 7 of Padovani et al. 2018 and Fig. 4 of IceCube Collaboration et al. 2018). This is confirmed also by its steep soft X-ray spectrum (e.g. Padovani \& Giommi 1996) and the SED upturn at $\approx 10^{18}$ $\mathrm{Hz}$.

The second clue comes from its optical spectrum (Paiano et al. 2018), which is characterized by a power-law continuum and, apart from faint interstellar features, displays three extremely weak emission lines identified as O II 3727 $\AA$, O III $5007 \AA$, and N II $6583 \AA$, with equivalent widths $\left(\mathrm{EWs}^{2}\right)$ ranging between 0.05 and $0.17 \AA$. TXS $0506+056$ therefore fully qualifies as a BL Lac according to the standard empirical definition (EW < 5 Å; Stickel et al. 1991; Stocke et al. 1991).

Why do BL Lacs have low EWs? Blandford \& Rees (1978) had originally suggested that the absence of emission lines in BL Lacs was due to a very bright, Doppler-boosted jet continuum, which was washing out the lines (said differently, the EW was low because the continuum was high; see also Georganopoulos \& Marscher 1998). In the years following that paper observations of various BL Lacs, mostly selected in the X-ray band, showed that in many cases their optical spectrum was not swamped by a non-thermal component, as host galaxy features were very visible (Stocke et al. 1991). It was then thought that most BL Lacs had intrinsically weak lines (i.e., the EW was low because the line was weak). To complicate matters some objects appeared to change class (e.g. Vermeulen et al. 1995; Pian et al. 1999). Giommi et al. $(2012,2013)$ have shown that these two possibilities are not exclusive and indeed are both viable. Therefore, objects so far classified as BL Lacs on the basis of their observed weak, or undetectable, emission lines belong to two physically different classes: intrinsically weak-lined objects, and heavily diluted broad-lined sources, which are in reality quasars. These latter objects have been labelled "masquerading BL Lacs" by Giommi et al. (2013). We stress that these sources typically have relatively high powers and $v_{\text {peak }}^{S}$ values (see Fig. 10 of Giommi et al. 2012), which translate into more non-thermal jet-related optical light than present in low $v_{\text {peak }}^{S}$ sources. This implies that the emission lines are

(IBL and HBL) sources respectively (Padovani \& Giommi 1995; Abdo et al. 2010).

2 The EW of a spectral line is a measure of its strength and it is (roughly) defined as its flux (in units of erg s $\mathrm{s}^{-1} \mathrm{~cm}^{-2}$ ) normalized by the continuum level underneath the line (in units of $\mathrm{erg} \mathrm{s}^{-1}$ $\left.\mathrm{cm}^{-2} \AA^{-1}\right)$. It is therefore measured in $\AA$.
Table 1. Masquerading BL Lacs ( $\equiv$ high $\nu_{\text {peak }}^{S}$ FSRQs): their properties in perspective.

\begin{tabular}{lccc} 
& BL Lacs & FSRQs & Masquerading BL Lacs \\
\hline accretion & inefficient & efficient & $\begin{array}{c}\text { efficient } \\
\text { (but apparently not) }\end{array}$ \\
$\mathrm{EW}$ & $<5 \AA$ & $>5 \AA$ & $<5 \AA$ \\
$L / L_{\mathrm{Edd}}$ & $\lesssim 0.01$ & $\gtrsim 0.01$ & $\gtrsim 0.01$ \\
$v_{\text {peak }}^{S}$ & any & $\lesssim 10^{14} \mathrm{~Hz}$ & $\gtrsim 10^{14} \mathrm{~Hz}$ \\
\hline
\end{tabular}

more easily diluted, explaining their BL Lac classification. In short, "masquerading BL Lacs" are the missing FSRQs with relatively high $v_{\text {peak }}^{S}$.

We stress that "real" BL Lacs and FSRQs belong to very different physical classes, namely objects without and with high-excitation emission lines in their optical spectra, referred to as low-excitation (LEGs) and high-excitation galaxies (HEGs), respectively. As discussed by Padovani et al. (2017) the LEG/HEG classification applies to AGN in general: quasars and Seyferts belong to the HEG category, while low-ionization nuclear emission-line regions (LINERs) and absorption line galaxies are classified as LEGs.

There are fundamental physical differences between these two types of AGN. Namely, LEGs exhibit radiatively inefficient accretion related to low Eddington ratio ${ }^{3}$ $\left(L / L_{\text {Edd }} \lesssim 0.01\right)$, while HEGs accrete in a radiatively efficient manner at high Eddington rates $\left(0.01 \lesssim L / L_{\text {Edd }} \lesssim 1^{4}\right.$; e.g. Padovani et al. 2017). From a theoretical perspective, the observed difference in $L / L_{\mathrm{Edd}}$ is generally associated with a switch between a standard accretion, i.e. radiatively efficient, geometrically thin (but optically thick) disk accretion flow (Shakura \& Sunyaev 1973) and a radiatively inefficient, geometrically thick (but optically thin) disk accretion flow (e.g. Narayan \& Yi 1995). Table 1 presents an overview of the properties of masquerading BL Lacs compared to those of real BL Lacs and FSRQs.

We contend that TXS $0506+056$ is a HEG and therefore a masquerading BL Lac, i.e., intrinsically an FSRQ with hidden broad lines and a standard Shakura - Sunyaev accretion disk. This claim is based on various pieces of evidence:

(i) Its radio power $\left(P_{1.4 \mathrm{GHz}} \sim 1.8 \times 10^{26} \mathrm{~W} \mathrm{~Hz}^{-1}\right)$ and $\mathrm{O}$ II luminosity $\left(L_{\mathrm{O}}\right.$ II $\sim 2 \times 10^{41} \mathrm{erg} \mathrm{s}^{-1}$ : Paiano et al. 2018) put this source right in the middle of the locus of jetted (radioloud) quasars (Fig. 4 of Kalfountzou et al. 2012). Moreover, HEGs become the dominant population in the radio sky above $P_{1.4 \mathrm{GHz}} \sim 10^{26} \mathrm{~W} \mathrm{~Hz}^{-1}$ (Heckman \& Best 2014);

\footnotetext{
3 This is the ratio between the (accretion-related) observed luminosity and the Eddington luminosity, $L_{\mathrm{Edd}}=1.26 \times 10^{46}\left(\mathrm{M} / 10^{8} \mathrm{M}_{\odot}\right)$ $\mathrm{erg} \mathrm{s}^{-1}$, where $\mathrm{M}_{\odot}$ is one solar mass. This is the maximum isotropic luminosity a body can achieve when there is balance between radiation pressure (on the electrons) and gravitational force (on the protons).

4 As stressed by Padovani et al. (2017) the dividing line in $L / L_{\text {Edd }}$ needs to be considered only in a statistical sense. The fundamental physical separation, in fact, may be also be a function of other parameters (such as spin and black hole mass) and in addition one should keep in mind that the observational data used to constrain this separation are subject to measurement and computational uncertainties and biases.
} 
(ii) The optical spectrum of TXS $0506+056$ resembles that of a Seyfert 2 galaxy from the point of view of the emission line ratios (Paiano et al. 2018), which implies it is a HEG;

(iii) Its $L / L_{\text {Edd }}$ is $>0.01$. We estimate the black hole mass by assuming the host galaxy to be a typical giant elliptical with absolute R-band magnitude $M(R) \sim-22.9$ (Paiano et al. 2018) and then by using the McLure \& Dunlop (2002) relationship between black hole mass and bulge $M(R)$ to derive $M_{\mathrm{BH}} \approx 3 \times 10^{8} M_{\odot}$. This translates into $L_{\mathrm{Edd}} \approx 4 \times 10^{46} \mathrm{erg}$ $\mathrm{s}^{-1}$. We note that the true value cannot be much larger than this. Paiano et al. (2018) have in fact estimated a lower limit on the redshift $>0.3$ based on the lack of absorption features due to the host galaxy. Given the closeness of this value to the measured redshift this means that the host galaxy can only be at the same level, or fainter, than assumed, which in turns means that the black hole mass cannot be much higher than estimated and that the resulting $L / L_{\text {Edd }}$ is a lower limit. As for the (thermal) bolometric luminosity, we use the relationships between $L_{\text {bol }}$ and $L_{\mathrm{O} \text { II }}$ and $L_{\mathrm{O} \text { III }}$ (Punsly \& Zhang 2011) to derive $L_{\text {bol }} \sim 9 \times 10^{45} \mathrm{erg} \mathrm{s}^{-1}$ and $L_{\text {bol }} \sim 3 \times 10^{45} \mathrm{erg} \mathrm{s}^{-1}$ respectively. Since Punsly \& Zhang (2011) have shown that these values are overestimated for jetted (radio-loud) quasars because of a sizeable jet-induced contribution we conservatively divide the logarithmic average by 3 (based on their Fig. 3), which gives $L_{\text {bol }} \sim 1.7 \times 10^{45}$ $\mathrm{erg} \mathrm{s}^{-1}$. We finally obtain $L / L_{\text {Edd }} \gtrsim 0.04$.

We can also estimate the broad-line region (BLR) luminosity in two different ways (top-down and bottom-up): 1 . assuming $\left\langle L_{\mathrm{bol}} / L_{\text {disk }}\right\rangle \approx 2$, which is consistent with typical quasar SEDs (e.g. Richards et al. 2006), we obtain $L_{\text {disk }} \sim 8 \times 10^{44} \mathrm{erg} \mathrm{s}^{-1}$, which translates into $L_{\mathrm{BLR}} \sim 8 \times 10^{43}$ $\mathrm{erg} \mathrm{s} \mathrm{s}^{-1}$ for a standard covering factor $\sim 10$ per cent; 2 . we derive the narrow line luminosity (NLR) from $L_{\mathrm{NLR}}=$ $3 \times\left(3 \times L_{\mathrm{O}}+1.5 \times L_{\mathrm{OIII}}\right) \sim 3 \times 10^{42} \mathrm{erg} \mathrm{s}^{-1}$ (Rawlings \& Saunders 1991), from which we get $L_{\mathrm{BLR}} \sim 3 \times 10^{43} \mathrm{erg}$ $\mathrm{s}^{-1}$ assuming $L_{\mathrm{BLR}} / L_{\mathrm{NLR}} \sim 10$, which is typical of FSRQs (Gu, Chen, \& Cao 2009). These give a logarithmic average $\sim 5 \times 10^{43} \mathrm{erg} \mathrm{s}^{-1}$.

Ghisellini et al. (2011) have proposed a classification scheme to divide BL Lacs from FSRQs, which is based on $L_{\mathrm{BLR}}$ in Eddington units, and set at a dividing value of $L_{\mathrm{BLR}} / L_{\mathrm{Edd}} \sim 5 \times 10^{-4}$, i.e. $L_{\text {disk }} / L_{\mathrm{Edd}} \sim 0.005$ (for a $\sim 10$ per cent covering factor). This turns out to be also the value, which separates radiatively efficient from radiatively inefficient regimes and in fact coincides with our LEG/HEG division (since $\left\langle L_{\mathrm{bol}} / L_{\mathrm{disk}}\right\rangle \approx 2$ ). In the case of TXS $0506+056$ $L_{\mathrm{BLR}} / L_{\mathrm{Edd}} \sim 0.001$, which implies that this source is an FSRQ also according to the Ghisellini et al. (2011) criterion. Sbarrato et al. (2012) have proposed a further division between BL Lacs and FSRQs at $L_{\gamma} / L_{\text {Edd }} \sim 0.1$. TXS 0506+056, with (an average) $L_{\gamma} / L_{\text {Edd }} \sim 0.7$, is well into the FRSQ region.

\section{TXS 0506+056 AS A "BLAZAR SEQUENCE" OUTLIER}

The existence of a strong anti-correlation between bolometric luminosity and $v_{\text {peak }}^{S}$, the so-called "blazar sequence", has been the subject of intense debate since it was first proposed

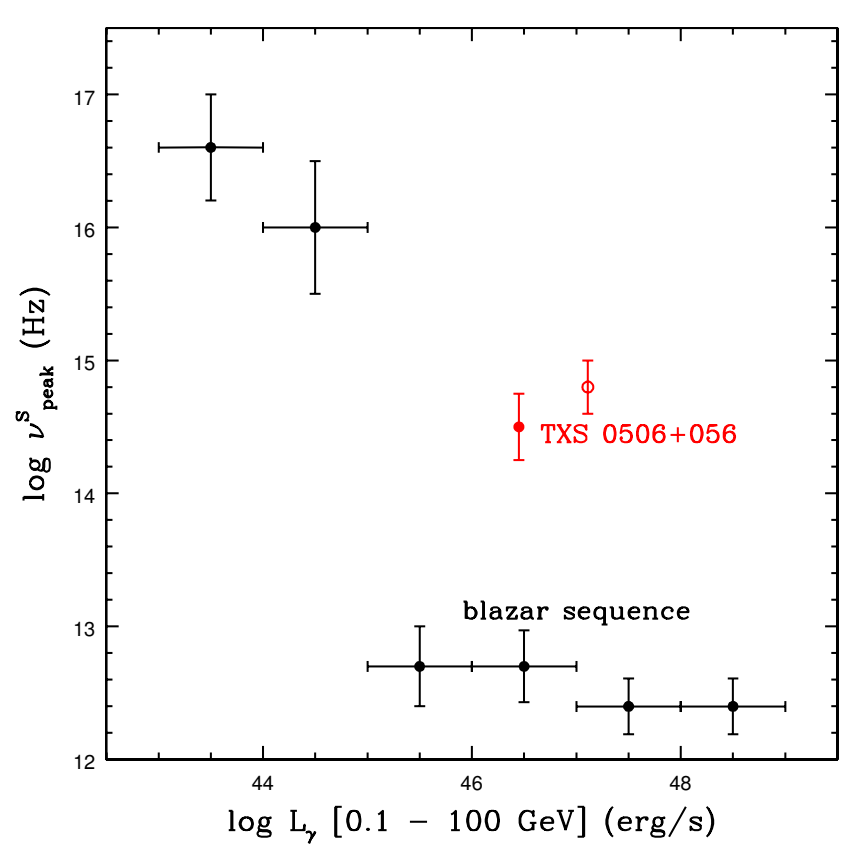

Figure 1. $v_{\text {peak }}^{S}$ versus $L_{\gamma}$ for the revised blazar sequence (black points; Ghisellini et al. 2017) and TXS 0506+056 (red points: average [filled] and $\gamma$-ray flare [open] values). Its $\gamma$-ray powers come from IceCube Collaboration et al. (2018), while the $v_{\text {peak }}^{S}$ values were derived by fitting the SED. The error bars denote the sample dispersion (blazar sequence) and the uncertainty (TXS 0506+056) respectively.

by Fossati et al. (1998) and Ghisellini et al. (1998) (see discussion and references in, e.g., Giommi et al. 2012). This is related to the apparent lack of FSRQs of the HBL type (Section 1). We do not wish here to (re-)enter this controversy but simply to look at things from the point of view of TXS 0506+056.

Many blazar sequence outliers have been discovered so far, both in the low power - low $v_{\text {peak }}^{S}$ (e.g. Padovani et al. 2003; Antón \& Browne 2005; Raiteri \& Capetti 2016) and high power - high $v_{\text {peak }}^{S}$ (e.g. Padovani, Giommi, \& Rau 2012; Kaur et al. 2017, 2018) regions of parameter space.

We have estimated $v_{\text {peak }}^{S}$ for TXS $0506+056$ by fitting both its average SED (using all available archival data: $v_{\text {peak }}^{S} \sim 10^{14.5 \pm 0.25} \mathrm{~Hz}$ ) and the one close to the time of the IceCube-170922A neutrino alert (Padovani et al. 2018, $v_{\text {peak }}^{S} \sim 10^{14.8 \pm 0.2} \mathrm{~Hz}$; see also Keivani et al. 2018, who find $v_{\text {peak }}^{S} \lesssim 10^{14.5} \mathrm{~Hz}$ ). Given its luminosities at various frequencies $\left(L_{\text {peak }} \sim 10^{46} \mathrm{erg} \mathrm{s}^{-1}\right.$, Section 2, and Padovani et al. 2018), TXS 0506+056 appears to be an outlier of the blazar sequence (see, e.g., Fig. 4 of Meyer et al. 2011, Fig. 10 of Giommi et al. 2012, and Fig. 6 of Padovani, Giommi, \& Rau 2012).

Ghisellini et al. (2017) have revisited the blazar sequence by using the Fermi 3LAC sample (Ackermann et al. 2015). $v_{\text {peak }}^{S}$ now changes quite abruptly as function of $L_{\gamma}(0.1-100 \mathrm{GeV})$, as shown in Fig. 1 (black points). The same figure shows also the location of TXS $0506+056$ in its 
average state (red filled symbol) and during the IceCube170922A neutrino alert (red open symbol). TXS 0506+056 is an obvious outlier even of the revised blazar sequence: given its $L_{\gamma}$, its $v_{\text {peak }}^{S}$ should be more than two orders of magnitude smaller to fit the sequence. We note that this is not unexpected, as masquerading BL Lacs have high powers and high $v_{\text {peak }}^{S}$ and therefore are by definition outliers.

Given that the first plausible high-energy neutrino source does not follow the blazar sequence, theoretical models predicting neutrino (and cosmic-ray) emission from blazars, which have the blazar sequence embedded in their calculations (e.g. Murase, Inoue, \& Dermer 2014; Rodrigues et al. 2018) will need to be revised.

\section{THEORETICAL IMPLICATIONS AND SUMMARY}

Using estimates of the $\gamma \gamma$ opacity on BLR photons and of the photopion $p \pi$ efficiency, we now discuss the implications of the presence of a BLR region on the location of the $\gamma$-ray emitting region and the neutrino output, assuming that $\gamma$ rays and neutrinos are produced in the same region of the jet.

The BLR photon field attenuates $\gamma$-rays of observed energy $E_{\gamma} \approx[25 \mathrm{GeV} /(1+z)]\left(\epsilon_{\mathrm{BLR}} / \epsilon_{\gamma}\right)$ (e.g., Ghisellini \& Tavecchio 2009; Sikora et al. 2009), where $\epsilon_{\mathrm{BLR}}=10.2 \mathrm{eV}$ is the energy of the $\operatorname{Ly} \alpha$ line, which makes the strongest contribution to the total BLR emission (quantities denoted with capital and lowercase letters refer to the observer and black-hole rest frame, respectively). The FSRQ nature of TXS 0506+056 also implies the existence of a dusty torus. However, attenuation of its infrared emission would become important only at $E_{\gamma} \gtrsim 1 \mathrm{TeV}$.

We thus calculate the optical depth for $\gamma$-rays on the BLR photons of TXS $0506+056$ as a function of distance of the emitting region, $R_{\mathrm{em}}$, from the central engine using the method of Böttcher \& Els (2016). We assume that the BLR is a spherical emitting shell with constant emissivity and width $0.2 R_{\mathrm{BLR}}$, extending from $0.8 R_{\mathrm{BLR}}$ to $R_{\mathrm{BLR}}$ (see Fig. 1 of Böttcher \& Els 2016). For the luminosity of the BLR we use the value derived in Section 2 . We estimate the radius of the BLR as $R_{\mathrm{BLR}} \simeq 10^{17} L_{\mathrm{d}, 45}^{1 / 2} \mathrm{~cm} \approx 7 \times 10^{16} \mathrm{~cm}$ (Ghisellini \& Tavecchio 2008), where $L_{\mathrm{d}, 45}=L_{\mathrm{disk}} /\left(10^{45} \mathrm{erg} \mathrm{s}^{-1}\right)$.

We model the radiation field of the BLR considering the 21 strongest lines from Francis et al. (1991). We find that for the luminosity derived in Section $2\left(L_{\mathrm{BLR}} \approx 5 \times 10^{43} \mathrm{erg} \mathrm{s}^{-1}\right)$, $\tau_{\mathrm{BLR}}\left(E_{\gamma}=100 \mathrm{GeV}\right) \approx 1$ at $R_{\mathrm{em}} \approx 0.9 R_{\mathrm{BLR}} \approx 6.5 \times 10^{16} \mathrm{~cm}$. The SED of TXS $0506+056$ as measured by MAGIC and VERITAS is consistent with significant absorption above $100 \mathrm{GeV}$ and $\tau_{\gamma \gamma}\left(E_{\gamma} \approx 100 \mathrm{GeV}\right) \approx 1$, when compared to the non strictly simultaneous Fermi-LAT data (Ansoldi et al. 2018; Abeysekara et al. 2018). This absorption is not related to the extragalactic background light, as the optical depth for $100 \mathrm{GeV}$ photons from a source at $z \approx 0.33$ is $\lesssim 0.1$. If the reported opacity is attributed to the BLR alone, then the $\gamma$ ray emitting region of TXS $0506+056$ should be located at its outer edge, i.e., $R_{\mathrm{em}} \gtrsim 0.9 R_{\mathrm{BLR}}$. Were the emission region closer to the central engine, stronger internal absorption of the $100 \mathrm{GeV} \gamma$-rays by the BLR would have been expected (e.g. Costamante et al. 2018). We note that for our assumed

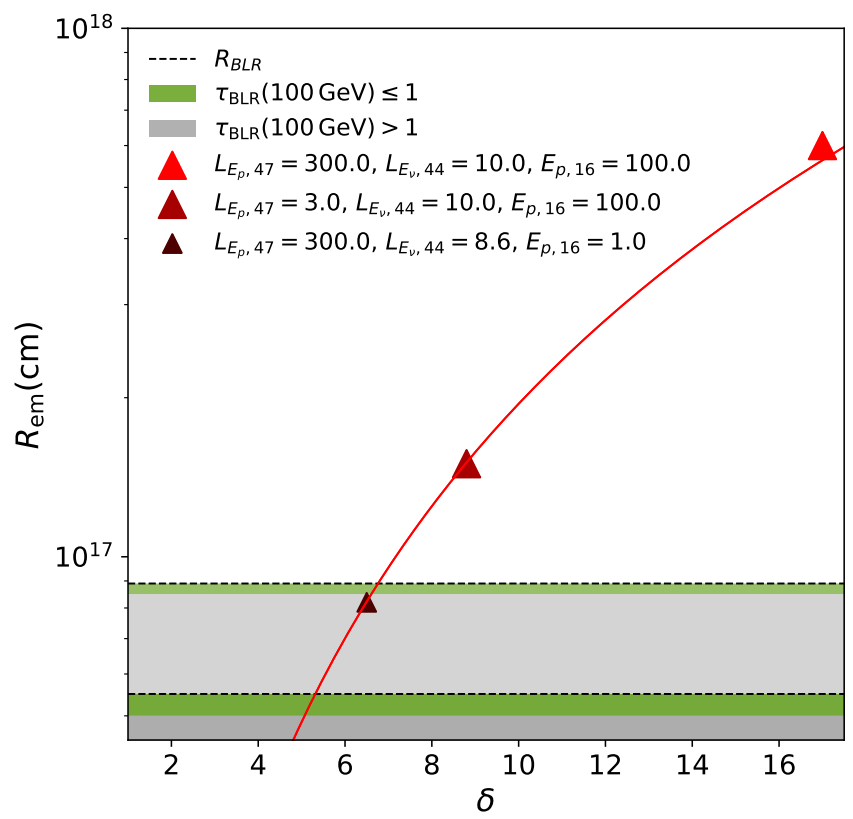

Figure 2. Location of the emitting region, $\boldsymbol{R}_{\mathrm{em}}$, along the jet as a function of the Doppler factor, $\delta$, for $t_{\mathrm{v}}=1$ day (red solid line) - see eq. (1). Dashed horizontal lines mark the values of the BLR radius that correspond to the range of $L_{\mathrm{BLR}}$ derived in Section 2. Green bands denote the respective ranges of $R_{\mathrm{em}}$, where $\tau_{\mathrm{BLR}}\left(E_{\gamma}=100 \mathrm{GeV}\right) \lesssim 1$, and grey bands regions where $\tau_{\mathrm{BLR}}\left(E_{\gamma}=\right.$ $100 \mathrm{GeV}$ ) $>1$. The minimum distance corresponding to $\delta_{\min }$ (see eq. (2)) is also indicated with red symbols for different parameters marked on the plot. The size of the symbols is proportional to the predicted all-flavour bolometric neutrino luminosity (see legend; where we used the notation $Q_{x}=Q / 10^{x}$ in $\mathrm{erg} \mathrm{s}^{-1}$, and $\mathrm{eV}$, for $L_{\mathrm{E}_{\mathrm{p}}}, L_{\mathrm{E}_{v}}$, and $E_{\mathrm{p}}$ respectively). If the blob lies within the BLR, then the constraint on $\delta_{\text {min }}$ (eq. (2)) must be modified to include interactions on BLR photons. Still, $E_{\nu} L_{\mathrm{E}_{\boldsymbol{v}}} \lesssim 10^{45} \mathrm{erg} \mathrm{s}^{-1}-$ see eq. (3).

dependence of $R_{\mathrm{BLR}}$ on $L_{\mathrm{disk}}$, the BLR energy density is independent of $L_{\text {disk }}$. Thus, varying $L_{\text {disk }}$ within the uncertainty range quoted in Section 2 changes the allowed range of $R_{\mathrm{em}}$ relative to $R_{\mathrm{BLR}}$ by $\lesssim 3$ per cent.

We can relate the radius of the emission region (blob), $R_{\mathrm{b}}^{\prime}$, to the distance of the dissipation region from the central engine $R_{\mathrm{em}}$, by assuming that the blob covers the crosssectional area of the jet:

$R_{\mathrm{em}}=\tan \theta_{j}^{-1} R_{\mathrm{b}}^{\prime} \approx \theta_{j}^{-1} R_{\mathrm{b}}^{\prime} \approx \Gamma R_{\mathrm{b}}^{\prime} \approx \delta^{2} c t_{\mathrm{v}}(1+z)^{-1}$,

where $\theta_{j} \approx 1 / \Gamma$ is the half-opening angle of a conical jet, $\Gamma$ is the Lorentz factor of the bulk flow, $\delta \approx \Gamma$ is the Doppler factor, $t_{\mathrm{V}}$ is the observed variability timescale, and primed variables denote quantities in the co-moving frame of the blob. For a fixed $t_{\mathrm{V}}$ (assumed equal to 1 day based on $\mathrm{X}$ ray variability: Keivani et al. 2018), different values of $\delta$ correspond to different locations according to eq. (1), as shown in Fig. 2 (red solid line). The range of $R_{\mathrm{em}}$ where $\tau_{\mathrm{BLR}}\left(E_{\gamma}=100 \mathrm{GeV}\right) \lesssim 1$ (green bands) lies within the outer radius of the BLR (dashed lines).

We next discuss constraints due to the electromagnetic 
(EM) cascade emission produced in the source from photohadronic $(p \gamma)$ interactions. We first derive constraints due to interactions with the blazar synchrotron photons (see also numerical results by Gao, Fedynitch, Winter \& Pohl 2018; Cerruti et al. 2019). We then discuss how the presence of the BLR changes these results.

We require that the luminosity of the photon component produced by $p \gamma$ interactions does not exceed the luminosity in the X-ray band $(0.3-10 \mathrm{keV})$, i.e. $L_{\mathrm{X}, \lim } \simeq$ $3 \times 10^{44} F_{\mathrm{X}, \mathrm{lim},-12} \mathrm{erg} \mathrm{s}^{-1}$ (IceCube Collaboration et al. 2018; Keivani et al. 2018). Using eqs. (9), (13), and (14) of Murase, Oikonomou, \& Petropoulou (2018) and assuming that a fraction $f_{x}$ of the bolometric cascade luminosity will emerge in the X-ray band, we derive a lower limit on the Doppler factor of the emitting region:

$$
\delta_{\text {min }}^{2+2 \beta} \approx f(\beta) \frac{3 \hat{\sigma}_{\mathrm{p} \pi} L_{\mathrm{s}}}{4 \pi c^{2} t_{\mathrm{v}} E_{\mathrm{S}}}\left[\frac{2 E_{\mathrm{p}} E_{\mathrm{S}}(1+z)^{2}}{m_{\mathrm{p}} c^{2} \bar{\epsilon}_{\Delta}}\right]^{\beta-1} \frac{E_{\mathrm{p}} L_{\mathrm{E}_{\mathrm{p}}}}{f_{x}^{-1} L_{\mathrm{X}, \mathrm{lim}}},
$$

where $\beta \sim 2.5$ is the photon index in the Swift/XRT energy band (IceCube Collaboration et al. 2018; Keivani et al. 2018), $f(\beta)=[2 /(1+\beta)][5 / 16+g(\beta) / 2], g(\beta) \simeq 0.01(30)^{\beta-1}$, $\hat{\sigma}_{\mathrm{p} \pi} \simeq 7 \times 10^{-29} \mathrm{~cm}^{2}, \bar{\epsilon}_{\Delta} \sim 0.3 \mathrm{GeV}, L_{\mathrm{s}} \sim 10^{46} \mathrm{erg} \mathrm{s}^{-1}$ and $E_{\mathrm{S}} \sim 1 \mathrm{eV}$ are the observed (isotropic) synchrotron luminosity and photon energy, respectively. The only free parameters in eq. (2) are the proton energy $E_{\mathrm{p}}$ and isotropic proton luminosity $E_{\mathrm{p}} L_{\mathrm{E}_{\mathrm{p}}}$. For $\delta=\delta_{\min }$ and $f_{x} \sim 0.1$ we obtain an upper limit on the all-flavour neutrino luminosity (e.g. Murase, Oikonomou, \& Petropoulou 2018):

$E_{v} L_{\mathrm{E}_{v}} \lesssim \frac{3}{8}\left[\frac{5}{16}+\frac{g(\beta)}{2}\right]^{-1} \frac{L_{\mathrm{X}, \lim }}{f_{x}} \approx 10^{45} \frac{L_{\mathrm{X}, \lim , 44.5}}{f_{x,-1}} \mathrm{erg} \mathrm{s}^{-1}$,

in agreement with detailed numerical modeling of the flaring SED of TXS 0506+056 (e.g. Keivani et al. 2018).

The location of a blob moving with $\delta_{\min }$ can be estimated by substitution of eq. (2) into eq. (1). The results obtained for different values of $E_{\mathrm{p}}$ and $E_{\mathrm{p}} L_{\mathrm{E}_{\mathrm{p}}}$ are presented in Fig. 2 (red symbols). We find that the blob is located beyond the BLR (dashed horizontal lines) unless $E_{\mathrm{p}} \leq 10 \mathrm{PeV}$ or $E_{\mathrm{p}} L_{\mathrm{E}_{\mathrm{p}}}<10^{47} \mathrm{erg} \mathrm{s}^{-1}$. In the latter case, the BLR provides additional target photons for $p \gamma$ interactions and one might expect that a higher neutrino luminosity can be achieved. However, the accompanying EM cascade component would be accordingly more luminous (see also Petropoulou \& Mastichiadis 2015). By requiring that the EM cascade emission from $p \gamma$ interactions on BLR photons does not overshoot the X-ray data (see also Petropoulou et al. 2017), we can also derive an upper bound on the expected $E_{v} L_{\mathrm{E}_{v}}$ from a blob located within the BLR, which is still given by eq. (3).

In conclusion, $\gamma \gamma$ opacity constraints allow the emitting region to be at the outer edge of the BLR (see Fig. 2), but the constraints from the cascade emission place an upper bound of $\sim 10^{45} \mathrm{erg} \mathrm{s}^{-1}$ to the all-flavour neutrino luminosity produced within a blob, irrespective of its location with respect to the BLR. We notice that the upper bound derived here is consistent with ${ }^{5}$, and well below, the neu-

5 In an ensemble of faint sources with a summed expectation of order 1 , one might observe a neutrino even if the individual expectation value is $\ll 1$ (IceCube Collaboration et al. 2018). Hence the upper limits. trino luminosity implied by the IceCube observations, assuming that the source was emitting neutrinos throughout the whole IceCube observation period of 7.5 years $\left(\lesssim 10^{46}\right.$ $\left.\mathrm{erg} \mathrm{s}^{-1}\right)$ and 6 months $\left(\lesssim 2 \times 10^{47} \mathrm{erg} \mathrm{s}^{-1}\right)$ respectively (IceCube Collaboration et al. 2018). The explanation of neutrino luminosities as high as $\sim 10^{47} \mathrm{erg} \mathrm{s}^{-1}$ as seen during the 2014-2015 neutrino flare (Padovani et al. 2018; IceCube Collaboration 2018) requires more complex theoretical scenarios that invoke more than one emitting region (e.g. Murase, Oikonomou, \& Petropoulou 2018).

In summary, the radio and O II luminosities, emission line ratios, and Eddington ratio of TXS 0506+056, all point to its re-classification as a masquerading BL Lac, namely an FSRQ with the emission lines heavily diluted by a strong, Doppler-boosted jet. Moreover, TXS 0506+056 has a $v_{\text {peak }}^{S}$, which is more than two orders of magnitude larger than expected by the blazar sequence. These two facts are likely to have an impact on the theoretical modeling of this source and on our understanding of neutrino emission in blazars.

\section{ACKNOWLEDGMENTS}

PP thanks the ASI Science Data Center (SSDC) for the hospitality and partial financial support for his visit. PG acknowledges the support of the TUM - IAS, funded by the German Excellence Initiative (and the European Union Seventh Framework Programme under grant agreement no. 291763). MP acknowledges support by the Lyman Jr. Spitzer Postdoctoral Fellowship. FO acknowledges useful corrrspondence and discussions with Markus Böttcher, Paul Els, Konstancja Satalecka, and Michael Unger. This work is supported by the Deutsche Forschungsgemeinschaft through grant SFB 1258 "Neutrinos and Dark Matter in Astro- and Particle Physics".

\section{REFERENCES}

Abdo A. A., Ackermann M., Ajello M., et al., 2010, ApJ, 716, 30 Abeysekara A. U., et al., 2018, ApJ, 861, L20

Ackermann M., et al., 2015, ApJ, 810, 14

Albert A., et al., 2018, ApJ, 863, L30

Ansoldi S., et al., 2018, ApJ, 863, L10

Antón S., Browne I. W. A., 2005, MNRAS, 356, 225

Blandford R. D., Rees M. J., 1978, in Pittsburg Conference on BL Lac Objects, Ed. A. M. Wolfe, Pittsburgh, University of Pittsburgh press, p. 328

Böttcher M., Els P., 2016, ApJ, 821, 102

Cerruti, M., Zech, A., Boisson, C., et al. 2019, MNRAS, 483, L12

Costamante L., Cutini S., Tosti G., Antolini E., Tramacere A., 2018, MNRAS, 477, 4749

Fossati G., Maraschi L., Celotti A., Comastri A., Ghisellini G., 1998 MNRAS, 299, 433

Francis P. J., Hewett P. C., Foltz C. B., Chaffee F. H., Weymann R. J., Morris S. L., 1991, ApJ, 373, 465

Gao S., Fedynitch A., Winter W., Pohl M., 2018, Nature Astronomy, 154

Georganopoulos M., Marscher A. P., 1998, ApJ, 506, 621

Ghisellini G., Celotti A., Fossati G., Maraschi L., Comastri A., 1998, MNRAS, 301, 451

Ghisellini G., Tavecchio F., 2008, MNRAS, 387, 1669

Ghisellini G., Tavecchio F., 2009, MNRAS, 397, 985

Ghisellini G., Tavecchio F., Foschini L., Ghirlanda G., 2011, MNRAS, 414, 2674 
Ghisellini G., Righi C., Costamante L., Tavecchio F., 2017, MNRAS, 469, 255

Giommi P., Padovani P., Polenta G., Turriziani S., D'Elia V., Piranomonte S., 2012, MNRAS, 420, 2899

Giommi P., Padovani P. \& Polenta G., 2013, MNRAS, 431, 1914

Gu M., Chen Z., Cao X., 2009, MNRAS, 397, 1705

Heckman T. M., Best P. N., 2014, ARA\&A, 52, 589

IceCube Collaboration, 2018, Science, 361, 147

IceCube Collaboration, Fermi-LAT, MAGIC, AGILE, ASASSN, HAWC, H.E.S.S., INTEGRAL, Kanata, Kiso, Kapteyn, Liverpool Telescope, Subaru, Swift/NuSTAR, VERITAS, VLA/17B-403 teams, 2018, Science, 361, eaat1378

Kalfountzou E., Jarvis M. J., Bonfield D. G., Hardcastle M. J., 2012, MNRAS, 427, 2401

Kaur A., et al., 2017, ApJ, 834, 41

Kaur A., Rau A., Ajello M., Domínguez A., Paliya V. S., Greiner J., Hartmann D. H., Schady P., 2018, ApJ, 859, 80

Keivani A., et al., 2018, ApJ, 864, 84

McLure R. J., Dunlop J. S., 2002, MNRAS, 331, 795

Meyer E. T., Fossati G., Georganopoulos M., Lister M. L., 2011, ApJ, 740, 98

Murase K., Inoue Y., Dermer C. D., 2014, PhRvD, 90, 023007

Murase K., Oikonomou F., Petropoulou M., 2018, ApJ, 865, 124

Narayan R., Yi I., 1995, ApJ, 452, 710

Padovani P., Giommi P., 1995, ApJ, 444, 567

Padovani P., Giommi P., 1996, MNRAS, 279, 526

Padovani P., Perlman E. S., Landt H., Giommi P., Perri M., 2003, ApJ, 588, 128

Padovani P., Giommi P., Rau A., 2012, MNRAS, 422, L48

Padovani P., et al., 2017, A\&ARv, 25, 2

Padovani P., Giommi P., Resconi E., T. Glauch, B. Arsioli, N. Sahakyan, M. Huber, 2018, MNRAS, 480, 192

Paiano S., Falomo R., Treves A., Scarpa R., 2018, ApJ, 854, L32

Petropoulou M., \& Mastichiadis A., 2015, MNRAS, 447, 36

Petropoulou M., Nalewajko K., Hayashida M., Mastichiadis A., 2017, MNRAS, 467, L16

Pian E., et al., 1999, ApJ, 521, 112

Punsly B., Zhang S., 2011, MNRAS, 412, L123

Raiteri C. M., Capetti A., 2016, A\&A, 587, A8

Rawlings S., Saunders R., 1991, Nature, 349, 138

Richards G. T., et al., 2006, ApJS, 166, 470

Rodrigues X., Fedynitch A., Gao S., Boncioli D., Winter W., 2018, ApJ, 854, 54

Sbarrato T., Ghisellini G., Maraschi L., Colpi M., 2012, MNRAS, 421,1764

Shakura N. I., Sunyaev R. A., 1973, A\&A, 24, 337

Sikora M., Stawarz Ł., Moderski R., Nalewajko K., Madejski G. M., 2009, ApJ, 704, 38

Stickel M., Padovani P., Urry C. M., Fried J. W., Kühr H., 1991, ApJ, 374, 431

Stocke J. T., Morris S. L., Gioia I. M., Maccacaro T., Schild R., Wolter A., Fleming T. A., Henry J. P., 1991, ApJS, 76, 813

Urry C. M., Padovani P., 1995, PASP, 107, 803

Vermeulen R. C., Ogle P. M., Tran H. D., Browne I. W. A., Cohen M. H., Readhead A. C. S., Taylor G. B., Goodrich R. W., 1995, ApJ, 452, L5

This paper has been typeset from a $\mathrm{T}_{\mathrm{E}} \mathrm{X} / \mathrm{IAT}_{\mathrm{E}} \mathrm{X}$ file prepared by the author. 